\title{
Hypothetical, not Fictional Worlds
}

\author{
Friedel Weinert \\ Professor of the Faculty of Social Sciences \\ University of Bradford, Bradford BD7 IDP, UK \\ f.weinert@bradford.ac.uk
}

\begin{abstract}
This paper critically analyzes the fiction-view of scientific modeling, which exploits presumed analogies between literary fiction and model building in science. The basic idea is that in both fiction and scientific modeling fictional worlds are created. The paper argues that the fiction-view comes closest to certain scientific thought experiments, especially those involving demons in science and to literary movements like naturalism. But the paper concludes that the dissimilarities prevail over the similarities. The fiction-view fails to do justice to the plurality of model types used in science; it fails to realize that a function like idealization only makes sense in science because models, unlike works of fiction, can be de-idealized; it fails to distinguish sufficiently between the make-believe (fictional) worlds created in fiction and the hypothetical (as-if) worlds envisaged in models. Representation characterized in the fiction-view as a license to draw inferences does not sufficiently distinguish between inferences in fiction from inferences in scientific modeling. To highlight the contrast the paper proposes to explicate representation in terms of satisfaction of constraints.
\end{abstract}

Keywords Abstraction, constraints, fiction-view, hypothetical worlds, idealization, models, representation.

DOI 10.1515/kjps-2016-0019

\section{Introduction}

It is commonly agreed today that scientific theories represent the external world via models, of which there are many different kinds. Theories are very general in nature, whilst models represent particular systems. There are also different accounts of 'representation' - from isomorphism 
and similarity to fit. In a recent development several writers (R. Frigg, P. Godfrey-Smith) have emphasized affinities between scientific modeling and literary fiction.

This new focus removes the representational force of models even further from previous accounts in terms of isomorphism and similarity. In this new approach, an argument by analogy is used to underline that both scientific models and literary fiction deal with 'imaginary worlds'. The claim is that this analogy can shed new light on the role of modeling in the natural sciences.

The purpose of this contribution is to critically analyze this thesis: a) by testing it against a form of model, which is not often discussed in the literature, but seems close to the new approach (i.e., the use of demons in scientific thought experiments); b) by comparing it with literary examples, in particular the literary movement of naturalism. Thought experiments involving demons are used in both science and philosophy as argument patterns to test the potential and limits of knowledge claims about the natural world. The paper will focus on Laplace's and Maxwell's demons. They present conceptual models, which allow their users to explore the coherence and consistency of accepted knowledge claims or to present bold new hypotheses, like Einstein's hypothesis of the equivalence of inertial and gravitational mass. Einstein used his celebrated elevator thought experiment to illustrate his discovery. The demons of science, as well as naturalism, suggest themselves for a critical analysis of the fiction-view, because of their apparent closeness to the fictional account. The paper will therefore start with a sympathetic approach to the fiction-view of scientific modeling and, like the role of demons in scientific thinking, explore its potential. The first task will be to understand the thesis, namely the assumed close relationship between scientific modeling and literary fiction. Although the fictional nature of the demons of science, and realistic works of fiction, seem to support this view, it turns out that there are significant differences between modeling in science and literary fiction. The proponents of the fiction-view tend to forget that there are no analogies without disanalogies. These disanalogies come to light with the notion of representation. Once these disanalogies are taken into account the similarities between these two 
activities - telling fictional stories and constructing scientific models are very superficial. The devil lies in the details.

\section{The Fiction-view of Scientific Modeling}

The fiction-view of scientific modeling consists of two parts: on the negative side it rejects semantic and structuralist views of modeling, with their tendency to emphasize relations like isomorphism and similarity between the model system and the target system. The latter is usually taken to be some aspect or part of the natural world. On the positive side it proposes to exploit analogies between model-systems and works of fiction. The central contention is that 'models are akin to places and characters of literary fictions', rather than, say, Vaihinger's broader notion of fiction. ${ }^{1}$

The analogy is based on the claim that model-systems are often treated 'as imagined concrete things of literary fiction.' ${ }^{2}$ The main motivation for the fiction-view is threefold, according to Giere ${ }^{3}$ :

a) Scientists sometimes invoke fictions, which envisage unrealizable or unrealized scenarios, as is certainly the case when demons are employed in scientific argumentation;

b) many scientific models are physically impossible to realize: think for instance of an idealized pendulum, friction-free surfaces in classical mechanics or indeed Einstein's elevator thought experiment;

c) theoretical models represent idealized entities. This type of model has a long history: consider for instance planetary models, whether of the geocentric or heliocentric type, which employ circular orbits and neglect planetary moons.

The fiction-view gains further support from the extensive use of idealizations and abstractions in scientific modeling. In abstraction, the human mind deliberately factors out certain parameters, which may have a measurable effect on the system under consideration. Thus Newton's

Frigg, 2010, 99; Fine, 1993

2 Godfrey-Smith, 2006, 736; cf. Godfrey-Smith, 2009, 91

3 Giere, 2008, §1; cf. Frigg, 2010, 100-103 
inverse square law of gravitation allows the computation of the gravitational attraction between two particular bodies (say the Earth and the moon) but the gravitational influence of all other celestial bodies on the Earth-moon system is deliberately neglected, even though it exists.

In idealization, inaccuracies and small deviations are 'straightened out' in order to arrive at a pure type, which may be easier to describe or compute. In many models of the solar system the orbit of planets is depicted as circular, even though it is elliptical to different degrees, because a circular orbit is easier to calculate than an elliptical one.

Due to these abstractions and idealizations models are 'at a remove' from the real target system. Hence it is tempting to say that they create possible worlds or imaginary systems, which are analogous to fictional accounts. Although scientific models are idealized systems, they nevertheless have the function of representing their target system. The proponents of the fiction-view understand representation in different ways: for instance indirect representation via similarity relations ${ }^{4}$ or as a license to draw 'inferences from the model to the target system.' ${ }^{5}$ One question, which arises, is whether this representational element is also present in literary fiction or whether the inferences are of the same type in both cases.

The proposed similarity between literary fiction and scientific models further implies that there is no clear distinction between fictional accounts, thought experiments - as for instance in the use of demons and scientific modeling. ${ }^{6}$ The reason for this fuzzy boundary between scientific models, literary accounts and thought experiments is that literary and non-literary thought experiments can be understood as conceptual models. As such they have many similarities with other models: they contain abstractions and idealizations and therefore describe 'fictional worlds'. But conceptual models in science also have representational functions because, like other scientific models, they aim at an investigation of real-world target systems.

\footnotetext{
$4 \quad$ Godfrey-Smith, 2006, Fn6

5 Frigg, 2010, Introduction

6 Frigg, 2010, 121-5
} 
In his analysis of the anatomy of scientific modeling, Frigg introduces a distinction between $p$-representation and $t$-representation.

$P$-representation ascribes properties to a fictional system. It is a description of an imaginary scenario, whereby a model system provides a description or attribution of fictional properties (but not, according to Frigg, fictional objects in order to avoid ontological commitments). This description should be 'understood by way of props in games of make-believe'. This function gains its credibility from the pretense theory of fiction. As a description is not inherently representational, a model must also be equipped with an additional representational function.

$T$-representation carries the representational force of the model. A model system denotes a target system. It incorporates a relation between the model system and the target system. Frigg uses a map analogy to characterize $t$-representation:

"The view that I am proposing is that one can think of a model-system as a kind of "generalized map" and explain how it represents (t-represents) its target along the lines of how maps represent their targets."

As an example Frigg uses the Newtonian model of the sun-Earth system. Its aim is to describe the Earth's orbit around the sun. However, given the huge number of different models in the natural and social sciences - from scale models, analogue models, conceptual models, functional models, hypothetical (as-if) models, ideal types, structural models - the question arises whether the map-analogy captures the essence of scientific modeling in general. Frigg claims that

"'model-systems are t-representations in the same way in which maps are: they denote a target system and certain facts obtain in them (...) which are then translated into claims about a target system by using a key." 8

In the case of scientific modelling two types of keys are highlighted.

\footnotetext{
$7 \quad$ Frigg, 2010, 100

8 Frigg, 2010, 128
} 
"The simplest of all keys is identity, the rule according to which facts in the model (or at least a suitably defined class of facts) are also facts in the world. For example, if $X$ t-represents $Y$ by identity, then it follows from the fact that $X$ has discrete energy levels that $Y$ has discrete energy levels too." 9

But identity has obvious limitations. A simple scale model of the solar system will represent the spatial distribution of the planets; it will often neglect its moons and present circular rather than elliptical orbits. It may not accurately represent the distances between the planets and their distance from the sun. Even a simple visible scale model will make abstractions from the real solar system and idealize their orbits to circular motion. The scale model is not a mirror image of the real system. A Newtonian model can also be a more abstract structural model, which combines algebraic and topological features, without being identical with the facts of the real system. The need for idealizations is stressed in the second example of a key.

"A more interesting key is the ideal limit key. Many model-systems are idealizations of the target in one way or another. A common kind of idealizations is to "push to the extreme" a property that a system possesses. This happens when we model particles as point masses, strings as massless, planets as spherical, and surfaces as frictionless." ${ }^{10}$

Many models $t$-represent their target systems not only via idealization but also by way of abstraction. The fundamental idea is that model-systems are built to $t$-represent target-systems. The emphasis on systems highlights a further function of models. Models typically select a limited number of parameters in order to represent a relationship between them, either spatial ordering (topological features as in planetary models) or mathematical ordering (algebraic features as in atom models). ${ }^{11}$ This fur-

9 Frigg, 2010, 131; italics in original

10 Frigg, 2010, 131; italics in original

11 On algebraic and topological features in models, see Weinert, 1999 
ther function - let's call it 'systematization' - is due to the fact that science deals with systems (rather than disparate facts) and that a system consists of a certain number of components and an interaction between them. Thus the solar system consists of nine planets (including Pluto) and the way the planets interact with each other and with the sun either through gravitation (Newton) or the curvature of space-time (Einstein).

A further important feature of scientific modeling is that these functions - abstraction, idealization, systematization - come in degrees, which means that features can be modified in more advanced models. This aspect of modeling will be called 'factualization'. The existence of these functions in scientific models plays an important part in the evaluation of the fiction-view.

The fiction analogy only strictly applies to $p$-representation, since it is doubtful, as will be argued below, that most fictional accounts have a representational function in the way that scientific models do. An analysis of the fiction-view of modeling must therefore operate on two levels.

The first question is whether there is a strong enough similarity between $p$-representation in literary fiction and scientific modeling to justify the label 'fiction-view' of scientific modeling. The second question breaks down into two parts: why $t$-representation, as it exists in scientific models, does not feature in fictional accounts (with some exceptions); and whether scientific modeling is comparable to a map, with a given key. One serious problem with the claim that 'models are akin to places and characters of literary fiction' is that it glosses over the numerous types of models, which exist in science and therefore cannot capture what is distinct about scientific models.

As a first approach one is struck by several disanalogies between scientific modeling and fictional accounts. It can be said that fictional accounts often describe an imaginary or possible world. Fiction tells stories, with evolving characters and plot lines; it creates characters and depicts situations. Scientific models lack these features. The difference between $p$-representation - the attribution of fictional attributes to the model and $t$-representation - the denotation of a relation between model-system and target system - suggests that a distinction should be made between the fictional worlds in literary texts and the hypothetical worlds described 
in scientific models. A fictional world is a make-believe possible world, the result of pure imagination. A hypothetical world is an as-if world, that is the model describes the world as if it consisted only of the components and relations included in the model. Hence it employs abstraction, idealization and the other functions. (As discussed below certain sophisticated models in science also provide explanation.)

Most literary works require an interpretation on the part of the reader or viewer. But there is no true interpretation or one key to unlock a literary text. Literary texts mostly lack the representational function of scientific models. This lack of a representational function also accounts for a disanalogy between literary text and maps. The former cannot be approached via a particular key, which is typical for maps. A key for literary texts would mean that the key opens the door to the one interpretation of the text (as is the case for maps). However, literary texts are typically open-ended and invite many different interpretations. Giere has pointed out that a failure of representation is a ground for criticism of a scientific model but not of a work of fiction. ${ }^{12}$

Frigg accepts that there are some 'salient differences' between literature and scientific modeling. ${ }^{13}$ Literary plots are complex, scientific modeling is often simple. Literary texts do not aim at a specific target system (with possibly one exception, as we shall see below). And aesthetic considerations play no role in scientific modeling.

The fact that fiction lacks a clear target system has a significant implication: a fictional world is a world of its own, in which there is no role for the intricate interplay between abstraction, idealization, systematization and factualization. This interplay means that many models are capable of de-idealization (or factualization). This particular feature is completely absent from fictional accounts.

Perhaps these disanalogies are just superficial differences. Perhaps the fiction-view is true of at least some scientific models, but not of others. To test the accuracy of the fiction-view, let us introduce some demons of science, as fictional characters; in particular Laplace's and Maxwell's

12 Giere, 2008, §3

13 Frigg, 2010, 125 
Demons. (See Weinert 2016) The French mathematician and physicist Pierre-Simon Laplace invoked the services of a superhuman being - a Demon - to investigate the properties of a deterministic scientific theory, like classical mechanics. To the Laplacean Demon, using the laws of classical physics, the whole universe appears like a long film strip, in which every frame, in the past, present and future, is already present.

In a famous thought experiment, involving 'a being with superior faculties', James Clerk Maxwell attempted to show that the Second law of thermodynamics only possessed statistical validity. This being, later dubbed 'Maxwell's Demon', is able to 'follow every molecule in its course'. In an appropriate setup such a being would be able, says Maxwell, to sort the molecules according to their respective velocities. The setup is simply a container, divided into two chambers by a partition, in which there is an opening. The Demon's only work involves the opening and closing of the hole so as to allow only the swifter molecules to pass from $A$ to $B$, and only the slower ones to pass from $B$ to $A$.

\section{Demons as Conceptual Models}

A focus on demons highlights several facts:

- Demons are conceptual models, which come closest to the idea of fiction. It has been suggested that all thought experiments are argument types. ${ }^{14}$ It is questionable whether such a claim is true in its generality but thought experiments, like those involving demons, do contain arguments regarding particular knowledge claims. At the same time one has to realize that conceptual models are only one type of model used in the natural and social sciences. There is of course no agreed category or classification of models in the sciences. It is unclear from the proponents' writings whether the fiction-view of models extends to all models in science. Consider, for instance, the functional model of demand and supply curves in economics, the analogue model of electric currents or as-if-models of planetary orbits - in all these cases it is difficult to see how the fiction-view can capture the essence of these models for they do not represent fictional worlds. It is more accurate to say that they

$\overline{14 \quad \text { Norton, } 1996}$ 
represent hypothetical scenarios. There is a difference between fictional worlds, which feature in literary works and hypothetical worlds, which feature in scientific models. Although the hypothetical worlds of scientific models are known to be non-existent, this feature is due to the departure - by degrees - from the real target systems, by way of abstraction, idealization etc. In fiction there are no calculated departures from the real world. On the other hand, there are certain types of literary fiction especially the movement of naturalism - which come closer in their characteristics to models in the sciences.

- Demons are hypothetical figures, only realizable in possible worlds; they only exist in our minds. They satisfy Giere's threefold motivation. Demons are therefore ideally placed to test the ideas behind the fiction-view of scientific modeling. Demons - like Laplace's or Maxwell's Demons - serve as thought experiments: their function is to test the coherence, consistency and extent of accepted knowledge claims, in this case classical physics.

Laplace employs his Demon to underline his view that the classical world is completely deterministic. Laplace bestows three valuable hypothetical attributes to his Demon: ${ }^{15}$

1. Computational omniscience, i.e. he is able to calculate both the past and future history of all systems - both micro- and macro-systems - from their current states of affairs.

The Demon is able to enjoy computational omniscience because he possesses a second attribute.

2. Dynamical omniscience, i.e. he is able to determine the true, not an estimated time evolution of the system under consideration. In the Demon's case ontological and predictive determinism coincide. The predictions he makes correspond precisely to the actual evolution of the system, the trajectory of which he calculates. There is no room for randomness.

Finally the Demon possesses:

15 Frigg et al., 2014 
3. Observational omniscience, i.e. he is not only able to determine the initial data of the current state of affairs from which he will compute both the past and the future. Laplace's Demon is a true inhabitant of the static Block Universe, since the whole vista of the course of the universe lies before his all-encompassing vision, like the frames of a film strip. Hence for the Demon Past, Present and Future seem equally real.

Thus $p$-representation is satisfied. What about $t$-representation? The Laplacean Demon $t$-represents a classical deterministic world. The Demon $t$-represents this classical world in an idealized way. (His representation is idealized because determinism does not strictly hold in classical physics, as is most famously illustrated by the three-body problem.) It is a hypothetical, not a fictional world because classical mechanics assumes that macro-systems exist in the real world and behave according to time-invariant deterministic laws.

Maxwell also bestows hypothetical properties on his Demon: he has the ability to sort slow from fast molecules. His aim is to disprove the original assumption that the Second law of thermodynamics is a deterministic law. The Demon opens a trapdoor between two chambers $A$ and $B$ and only lets one type of molecule, say, fast ones enter chamber $A$. In this way the Demon heats up chamber $A$ and cools chamber $B$. Most significantly Maxwell's Demon sorts out the molecules without expenditure of energy (or so Maxwell claims) and thus achieves a violation of the Second law of thermodynamics, as it was understood in the 19th century (as a deterministic law). Again $p$-representation is satisfied and so is t-representation. Maxwell's Demon represents an idealized thermodynamic gas system. It is a hypothetical, not a fictional world because statistical mechanics assumes that micro-systems exist in the real world and behave according to time-invariant deterministic laws.

Although the Demons are idealizations, the intention of their creators is not to tell a story about a possible, if fictional world. The intention is to $t$-represent the real world: either the deterministic world of classical mechanics (Laplacean Demon) or the probabilistic world of statistical mechanics (Maxwell's Demon). Novels and plays also denote possible worlds but they are particular fictional worlds, which mostly lack a particular target system. Yet exceptions exist. There are realistic novels or 
plays, especially belonging to the literary movement of naturalism, which represent 'real' society. Historical novels belong to this category. Their fictional characters may be said to be idealizations. But they still lack a specific feature of scientific models - namely the interplay of abstraction, idealization, systematization and factualization. An important aspect in scientific modeling is that these functions come in degrees. Hence abstraction can be removed, idealization can be factualized, and systematization can be improved, making the model more realistic. Thus an analysis of classical physics has shown that it is not truly deterministic, in the Laplacean sense; and an analysis of statistical mechanics has shown that the Maxwellian Demon must fail in his task of separating the fast from the slow molecules. The Demon himself is subject to the Second law.

Thus we have considered the fiction-view both from the point of view of the demons of science and from the perspective of (realistic) fiction. Despite first impressions, demons, as conceptual models, do not really support the fiction-view of models, unless that view simply claims that scientific models often represent idealized worlds. But the idealized worlds of fictional accounts and scientific models are not the same worlds. Although the demons of science are conceptual models, which seem to approximate the fiction-view, they display both $p$-representation and $t$-representation. They represent hypothetical but not (purely) fictional worlds. Fiction, in general, has no particular target system in the real world. It depicts imaginary worlds, perhaps inspired by the real world. But realistic novels and plays can be said to have generalized target systems, like society or historical epochs. These types of conceptual models are more likely to support the fiction-view. Still, differences persist. The differences between fictional worlds and hypothetical worlds become clearer when we turn to the question of representation, according to which models connect theory with reality. 


\section{Representation}

Frigg characterizes representation as a license to draw inferences from a model to a target system. ${ }^{16}$ Suárez, too, describes representation as surrogate reasoning: infer some features of $B$ (target system) by investigating $A$ (source). ${ }^{17}$ But model construction can only occur when some information about the target system is available, either of a theoretical or empirical kind. As discussed below, atom models were constructed in order to make sense of the experimental observations about them. Once the models were available - Thomson's plum pudding model and Rutherford's nucleus model - it became possible to draw inferences about the target systems. Rutherford effectively inferred from his scattering experiments that the plum pudding model was mistaken, since it could not account for the scattering of a-particles. Such inferences can go astray: the Greeks knew the orbital periods of the 6 known planets of antiquity and their order, with one exception: from their observations they placed the Earth at the centre and made the sun the third planet of their geocentric world view. Although the Greeks knew little about the planetary system their limited observational knowledge still acted as a constraint on their modeling exercises.

If models involve the drawing of inferences, namely from the model to the target system, then modeling involves the notion of representation. But in a scientific context more is needed than a license to infer. For a license to infer also exists in the fictional context: a reader of a novel, the spectator of a play, is licensed to infer certain lessons about what message the novel or the play is intended to convey. The reader or spectator is invited to interpret the work of art. Take, for instance, Thornton Wilder's play Our Town (1938). It is a play about the humdrum existence of people in a small American community. They follow their daily routines without much thought to the higher things in life. There are unfulfilled aspirations, like a trip to Paris. The characters go through the cycles of life - from love to loss, from little pleasures to great tragedies - as could be observed in any community. The twist of the play comes when

\footnotetext{
16 Frigg, 2010, 98
}

17 Suarez, 2003, §3 
some of the town characters die and are seen to gather in the afterworld from which they observe the goings-on in the town below on Earth. They observe proceedings with much incomprehension. Their most striking comment is: 'they do not understand!' As spectators we are licensed to infer a lesson about life: that often we do not see the wood for the trees; that we get lost in petty details without realizing that there may be higher aspirations in life, grander views to contemplate. That is, we are licensed to interpret the message of the play, which may not be identical with the intention of the author. Such an interpretation may not be shared by other viewers or they may put the emphasis on other features of the play. A literary text is deliberately open-ended; it is an invitation to the readers or spectators to make their own sense of proceedings.

What is different in scientific representation is that it requires a certain amount of objectivity. How can we capture this objectivity, which acts as a constraint on model building? A must have an underlying structure which allows agents to draw specific inferences about $B .{ }^{18}$ According to Frigg, models represent phenomena since 'science is about phenomena'. ${ }^{19}$ These phenomena are represented in models, and we are to think of models as 'generalized maps'. This leads to a two-fold ambiguity of the fiction-view. On the one hand we are to think of models as 'akin to places and characters in fiction'; on the other hand, we are to think of models as 'generalized maps'. Maps come with particular keys but literary texts are open-ended. It is this open-endedness which makes them unable to provide a particular key of how to interpret them. Maps do come with particular keys and do represent particular target systems. They are not open-ended. But scientific models, although they represent particular target systems, lack particular keys: neither 'identity' nor 'idealizations' are precise keys and they are not exhaustive.

Unlike Thornton Wilder's fictional town, a 'map' aims to represent a real system: an underground map of a big city represents, in an idealized fashion, not only the train lines and their terminals but the individual stations at which the trains will stop, the geographic direction of the

18 Suárez, 2004, §3

19 Frigg, 2010, 110 
line (from east to west, from north to south) and the interchanges. That is the maps of the underground trains are much more constrained than Thornton Wilder's fictional account of his 'little town'. Maps can also be improved or 'factualized', unlike fictional works.

As mentioned above, Frigg speaks of identity and ideal limits as examples of keys in scientific models. These keys are mere hypothesis and according to Frigg, little work has been done on keys. Therefore, Frigg soon distances himself from this thesis: scientific representation rarely comes with a key. But is a key needed to relate certain properties of the model to properties of the target system? R. Giere has introduced the notion of 'fit' into the discussion to capture the representational relation between model and world.

It is not the model that is doing the representing; it is the scientist using the model who is doing the representing. One way scientists do this is by picking out some specific features of the model that are then claimed to be similar to features of the designated real system to some (perhaps fairly loosely indicated) degree of fit. It is the existence of the specified similarities that makes possible the use of the model to represent the real system in this way. ${ }^{20}$

Giere describes 'fit', in an unspecified sense, as agreement with data. That is, claims about 'good fit' do not function as fictitious claims. ${ }^{21}$ Giere still envisages a loose sense of similarity as the relation between models and the world. But we should take into consideration that notions like isomorphism and similarity do not really explain the representation of reality by a model. ${ }^{22}$ Suárez has shown that both similarity and isomorphism do not possess the logical properties of representation. Representation is non-symmetric, non-transitive and non-reflexive. But similarity is reflexive and symmetric and isomorphism is reflexive, systematic and transitive. Furthermore isomorphism (the structure of $A$ is isomorphic to the structure of $B$ ) cannot account for inaccurate representation, whilst similarity cannot account for idealized representation. The demons do

20 Giere, 2004, §4.1; cf. Bailer-Jones, 2003

21 Giere, 2008, §3

22 Suárez, 2003; 2004; 2010;cf. Frigg, 2010 
not represent their target system in the sense of isomorphism or similarity. In fact, isomorphism and similarity are neither necessary nor sufficient for representation. One should add that notions like isomorphism and similarity are either used as primitives or fail to specify the degrees of abstraction, idealization and factualization, which clearly operate in the case of models.

Thus the notions of 'fit' (Giere) or 'key' (Frigg) are still underdeveloped. But Giere also observes that scientific principles and specific conditions constrain the structure of models. In what follows I propose to elaborate and explicate the notions of 'fit' and 'key' in terms of 'satisfaction of constraints'. The constraints set up a constraint space into which models try to fit. Fit means that the model structure successfully accommodates the available constraints. ${ }^{23} \mathrm{Fit}$, in terms of constraints, secures the objectivity involved in scientific models.

First, constraints will be understood generally as restrictive conditions on admissibility. Admittance to certain clubs carries age or even gender limits. Admissible are only those who satisfy these particular constraints. Similarly, constraints operate on scientific models and theories, which limit the admissible input of data. They create 'logical spaces', in which models are to be accommodated.

We can distinguish between empirical constraints, theoretical constraints and metaphysical constraints. Empirical constraints consist of (partial) data consistency, fundamental physical constants, and empirical laws. Theoretical constraints consist of internal consistency, external coherence, mathematical requirements (like differentiability or discreteness) and methodological norms (like testability). Einstein, for instance, imposed four constraints on scientific theories:

- The invariance of the velocity of light, $c$, in vacuum;

- The Special and General Relativity principles;

- Invariance and symmetries;

- Covariance (or form invariance).

23 Weinert 2000; Weinert 2006; cf. Bailer-Jones, 2009, Ch. VI, VIII 
The invariance of the velocity of light was at first simply an empirical constraint but Einstein turned it into a postulate of the Special theory of relativity. Metaphysical constraints, as conveyed by Laplace's and Maxwell's Demons, consist of fundamental views about nature, like determinism or indeterminism, continuity or discontinuity in nature. As constraints change over time, different constraint structures give rise to different logical spaces. It should be immediately obvious that fictional accounts satisfy far fewer constraints than scientific models. One expects fiction to be internally coherent in the development of the narrative, although even this constraint may be violated. There is no limitation on fictional accounts to respect the constraints of physical possibility. For instance, in the film Avatar the image of floating mountains takes the viewers by surprise. Note that Laplace's and Maxwell's Demons do not violate the laws of physical possibility.

Fit comes in degrees because the constraint space can be manipulated by introducing further or more specific constraints. But the more constraints a model satisfies the better is its chance of representational accuracy. Fit means that the model structure successfully accommodates the empirical and theoretical constraints. Models, like the demons, often reflect metaphysical constraints. Constraints should enhance a model's representational force. But an important requirement is that the constraints themselves must be accurate. How this particular feature works differentially can be gleaned, as discussed below, from the contrast between the history of geocentrism and the evolution of atom models. Fit also has a pragmatic dimension. It is dependent on the availability of constraints and their interplay. Consider, for instance, the Laplacean Demon. As a conceptual model, the Demon represents a completely deterministic classical world. The Laplacean Demon can be thought of as a superhuman scientist who can explore the resources of classical physics to such an extent that he uses it as a deterministic theory representing a presumably deterministic world. As it turns out, however, when the limits of classical physics are explored it is not as deterministic as the Laplacean Demon pretends. When the constraints are tightened and they themselves attain empirical and theoretical validity, a model which accommodates the constraints, is likely to be a good representation of the target system. 
This tightening of the constraint space is absent from fictional accounts. Novels, plays are taken at face value - it is not part of the interpretation whether they accurately represent a target system. What matters is their internal consistency and the depiction of the characters. As mentioned above, Thornton Wilder's Our Town tells the story of the fictional American small town of Grover's Corners between 1901 and 1913 through the everyday lives of its citizens. It is not part of the interpretation of Wilder's fictional town to ask whether it accurately represents an American town. It could depict any town, at least in the Western World. The only question, which is asked, is about the internal consistency of the character development. This facet is even more striking in the case of very abstract plays, like Beckett's Happy Days (1960). The play has two characters, Winnie and her husband Willie. In Act I, Winnie is embedded waist-deep in a low mound under blazing light, with a large black bag beside her. She is woken by a piercing bell, and begins her daily routine with a prayer. She prattles incessantly to her husband Willie, who is hidden from view behind the mound and is mostly taciturn. In Act II Winnie's situation seems to have worsened since she has now sunk up to her neck in the mound. But she does not seem to realize or to mind, since the incessant flow of her words does not stop or even decelerate. The play depicts a situation, which the viewer is invited to interpret. But it does not represent a particular target system; it does not provide a key. Winnie and Willie do not represent real people, their mound does not represent a real home. The play is about a message, not about an accurate or inaccurate representation of a target system.

The constraint space, which operates on scientific models, covers both what Frigg calls $p$-representation and $t$-representation. When proponents of the fiction-view suggest that a model-system describes fictional characteristics, their statement hides an ambiguity. Do they mean 'fictional' in the sense of an imaginary, make-believe world or do they mean an as-if, 'hypothetical' world in the sense of abstraction, idealization, systematization and factualization? The fiction-view conflates this distinction. Whilst Laplace's and Maxwell's Demons possess superhuman characteristics - the ability to predict and retrodict all micro- and macro-events of the classical world or the ability to sort molecules at will according to their speed - these characteristics are not purely fictional; 
they are idealizations of human predictive and manipulative capacities. Similar constraints operate on $t$-representation because, by definition, it spells out a relation between the model system and the target system. A good model seeks a close fit to the target system by satisfying a number of constraints, as a brief excursion into the history of atom models will show below. Such constraints do not generally operate on fictional accounts.

There are certain literary movements - in particular naturalism at the end of the 19th century with representatives like E. Zola and G. Hauptmann - which come closer to the fiction-view of scientific modeling than other literary genres. Naturalistic novels and plays use detailed realism to expose the hardship of life, including poverty, violence and disease, of the lower classes. Such naturalistic works of art resemble scientific models in that they ascribe certain realistic characteristics to the characters who, in the author's view, live in unacceptable social conditions. Hauptmann's play The Weavers (1892) portrays a group of Silesian weavers who stage an uprising in 1844 to protest against the exploitation and inhumane treatment in the wake of the Industrial Revolution. The play has a concrete target system, namely the life of a community of weavers in a particular part of Germany but it is not a socio-economic history of that community. Naturalism imposes more constraints on itself than do other literary movements. Scientific models do not have the liberty to dispense with constraints. At the opposite end of naturalism stands the work of a writer like Samuel Beckett whose novels and plays belong to the movement of the absurd. His characters have imaginary and unrealistic features, with no pretence that they may depict reality. Nevertheless, they contain messages about reality, which the viewer must infer in an act of interpretation. Perhaps the message of Happy Days - but this is an interpretation - is that humans gloss over the absurdity of the human condition by trivial small talk.

To say that scientific models are analogous to fiction is to neglect that where there is an analogy there is also a disanalogy. If fit is understood in terms of satisfaction of constraints it becomes clear that most fictional accounts are subject to very few constraints. But in order to secure a representation of a target system by a model system, in order to make representation successful, a model system is subject to a num- 
ber of constraints. These constraints are of course flexible and change over time. With the introduction of new constraints a model can improve its representation. For instance one of the major preconditions for the success of heliocentrism was the replacement of the Aristotelian theory of motion by the medieval impetus theory of motion. The impetus theory allowed the nominalist philosophers at the University of Paris, and indeed Copernicus, to parry some of the major Greek objections against a moving Earth. They were based on the Aristotelian theory of motion, according to which everything that moves needs a mover; and material objects like the Earth have a natural place (the centre) to which they would strive to return if they were removed from it. The Aristotelian theory of motion had allowed the Greeks to reject the notion of a moving Earth. For if the Earth moved, contrary to its natural rest, violent winds would blow from east to west, buildings would crumble and birds would never be seen to fly from west to east. Also objects would not fall straight down to the bottom of a tower. As none of these things were observed, the Greeks concluded that the Earth was stationary. The impetus theory allowed the medieval scholastics to point out that the atmosphere, the tower, all earthly objects moved along with a naturally rotating Earth. The Earth thus had a natural impetus which bestowed, as we would say today, inertial motion on the physical systems on the surface of the Earth. This shows that if a model is based on inaccurate constraints - like the Aristotelian theory of motion - its representational accuracy will suffer.

The history of atom models reveals the opposite effect, namely how improved constraints lead to better models of the atom. The case of atom models confirms that representation can only be achieved if the model builder already possesses some knowledge of the target system. Important models of the constitution of the atom were introduced between 1897 - the year Thomson discovered the electron - and 1911, when Rutherford inferred the existence of atomic nuclei from the experimental evidence of the so-called scattering experiments. The atom models ranged from poly-electron models to pair models, from Nagaoka's Saturnian model to Rutherford's nucleus model. ${ }^{24}$ It is important to realise that efforts to build atom models of increasing agreement with experimental

24 See Heilbron, 1981; Pais, 1986, Ch. 9; Weinert, 2000 
data were driven by the need to interpret the mounting experimental data. That is, the models were inferred from the experimental data, with little theoretical guidance, since there was no viable theory prior to the emergence of the new quantum mechanics in 1925.25 The two types of atom models, which will be of particular importance for the discussion in this paper, are Thomson's plum pudding model and Rutherford's nucleus model. Rutherford's model of the atom succeeded Thomson's in more than a chronological sense. It was Rutherford's use of an inference to the most likely explanation, which put an end to the career of the plum pudding model. It will be useful to characterise these two models briefly.

- Thomson's atom model had electrons but no nucleus. The electrons were arranged in rings, embedded in a positive sphere of electrification. (Analogy: the electrons are like plums swimming in a custard sauce.) But for the sake of stability, this model must be dynamic. The electrons move in rings at an angular velocity, w. As we go from the inside of the sphere outwards, the number of electrons on the rings increases. There is a spatial order among the rings to guarantee the stability of the atom. The Thomson model was able to cut the number of electrons, which were supposed to exist in the atom. Early speculations had placed thousands of electrons even in the hydrogen atom. By relating the number of electrons, $n$, in an atom to the atomic weight, $A$, Thomson drastically reduced the number of electrons, arriving at approximately one for the hydrogen atom. It turned out to be one of the reasons for the demise of the plum pudding model that the number of electrons in an atom is not determined by the atomic weight, $A$, but by the atomic number $Z$. It was the strength of the Rutherford model that it could account for this relationship.

- The striking feature of the Rutherford model (1911) was that it acquired a nucleus. This was dictated by experiment. Rutherford worried little about the electrons in the atom, because due to their small mass, they could not be responsible for the scattering, in some cases spectacular, which had been observed. In these scattering experiments, a-particles (helium atoms stripped of their 2 electrons) were fired at atoms

25 Born, 1949, 86 
in a thin layer of gold. All kinds of scattering angles were observed, but the most surprising result was that 1 in 8000 a-particles suffered deflections of more than ninety degrees. This was difficult to accommodate on the Thomson model. Rutherford explained that 'the mass, momentum and kinetic energy of the a-projectile (were) very large compared with the corresponding value of the electron in rapid motion.' 26 It was difficult to imagine that the electrons could deflect the massive a-particles. By contrast, if the atom acquired a nucleus, with a strong central charge, the deflection could easily be explained. If the a-particle, with positive charge, passes through the charged field of the nucleus, it will be deflected at an angle, which depends on the closeness of the approach to the nucleus. Using these results, and the relationship between $n$ and $Z$, Rutherford used said inference to discredit the Thomson model. Note that in the absence of a theory of atoms, Rutherford's nucleus model provided an explanation of large angle-scattering.

Whilst scientific models undergo change (they can be discarded - like the geocentric model - or improved - like atom models) as a result of the interplay of constraints, this is not the case with fictional accounts. It is a legitimate objection against scientific models that they disregard certain constraints, which can lead to their abandonment. But the excessive abstraction of Beckett's plays and novels is not a valid objection against their literary value. Berthold Brecht's play The Life of Galileo (1945-47) is a fictionalized yet realistic account of Galileo's struggle with the Catholic Church. Even Brecht's play about Galileo gives a very biased account of the behaviour of the Catholic Church and their representatives. They are depicted as the forces of reaction and orthodoxy throwing spanners in the wheels of the enlightened Galileo. The reality was more complex. A scientific model will be criticized for the poor representation of its target system; a work of fiction will at best be criticized for the poor execution of its plot and delineation of its characters.

26 Rutherford, 1911, 252-3 


\section{Analogies and Disanalogies}

The fiction-view relies on the existence of certain analogies between fiction and scientific models, especially the attribution of imaginary characteristics, which in the case of scientific models is due to the role of abstraction and idealization. A literary movement like naturalism comes closest to the role of scientific modeling but it is only one of many literary movements. The analogies are outweighed by the disanalogies, which come to light when the many different types of scientific models are taken into consideration. Works of fiction are final but scientific models undergo modification; the introduction of new constraints can lead to their refinement, as for instance in the case of atom models. De-idealization is an important feature of scientific modeling but it is absent from literary fiction. The constraint structure is very different in each case: most fictional works are only subject to a few internal constraints, like logical consistency, but most scientific models are subject to both internal constraints (like logical consistency and mathematical requirements) and external constraints (like coherence and testability). With a few exceptions, like the literary movement of naturalism, literary works of art fail to represent a clear, specific target system. But in the absence of clear target systems, it is impossible - as is the case with literary fiction - to assess degrees of abstraction and idealization, hence the extent of factualization, which operates in the case of many scientific models. Most planetary models assume circular orbits because a circular orbit is easier to compute than an ellipsis. But it is well known how much particular planets deviate from circularity: The eccentricity of the Earth's orbit is currently about 0.0167 , which makes the Earth's orbit nearly circular. Mercury has the greatest orbital eccentricity of any planet in the Solar System $(e=0.2056)$. By contrast it makes little sense to ask by how much Beckett's, Hauptmann's or Wilder's plays deviate from 'reality'.

The ascription of certain imaginary, fictional or unrealistic features $p$-representation - which seems to be present in both scientific models and literary fiction invites the analogy, on which the fiction-view of scientific modeling is based. But this similarity is very superficial. In scientific models we observe a depiction of hypothetical scenarios, with an interplay of abstraction, idealization, systematization and factualization, 
which make scientific models much more adaptable than literary fiction. In literary fiction there simply is no such interplay: works of fiction are not subject to the introduction or the refinement of newer constraints. Very few restrictive conditions operate on literary fiction - they are not accommodated in well-defined constraint spaces. The only valid similarity between works of fiction and models in science is their use of 'imagined concrete things'. But the similarity of this function glosses over a distinction between fictional features in literary works and hypothetical features in scientific models.

In most literary works of art there is no $t$-representation, as required in scientific models, let alone a key to the interpretation of the text. In the case of scientific models it is equally doubtful whether there is a particular key, as Frigg admits, to their representational function. The reason is the number of models, which are in circulation in science. A scale model of the planetary system does not $t$-represent in the same way, as say, a functional supply-and-demand model in economics. This paper has proposed that notions like fit and 'key' can be expressed in terms of the constraints, which the models must satisfy. Frigg envisages two keys: identity and ideal limits. If identity is required - which Frigg describes as the agreement of certain facts about the models, say discreteness in atom models, with facts about atoms - then this limited identity can be expressed in terms of the constraints, which are imposed on the model. (This 'identity' cannot be complete because of the unavoidable idealization in models.) Scale models tend to agree with certain features of the target system - the scale model of a bridge or the solar system shares topological features with the target system - but the notion of identity fails to capture the degree, to which the model 'resembles' the target system. A scale model of the solar system can be made more precise by the addition of further constraints: the inclusion of moons, the inclusion of all the known planets but it cannot properly capture the 'distance-orbit' relationship. The notion of constraint also expresses the 'key' of ideal limits better: for instance, the ideal pendulum can be shown to be a limit of the physical pendulum by abstracting from certain features: the period of an ideal pendulum - - is only dependent on the length of the string, $l$, and the gravitational constant, $g$, but not on its mass. The ideal pendulum, which is a model of a real pendulum, abstracts from 
the mass of the oscillating bob (and the string), and idealizes the string to a weightless, inextensible cord of length, I. These abstractions and idealizations can be lifted to make the model more realistic.

Finally, the fiction-view is a hybrid: it emphasizes the attribution of fictional characteristics, from which it derives its name. It does not sufficiently distinguish between imaginary characteristics, which are purely fictional, and hypothetical characteristics, which are abstracted or idealized from real target systems. But it cannot neglect the representational function of models, which are absent from (most) fictional works of art. So it introduces the map analogy. But maps usually have just one key, whilst scientific models are said to exhibit the example keys of 'identity' and 'ideal limit'. But in the language of constraints, models have many 'keys' but keys do not possess the adaptability of constraints. The structure of specific target systems acts as an important constraint on the objectivity of models in science but no literary critic would criticize the lack of objectivity in, say, Beckett's plays. However, if naturalistic novels or plays were to depart too much from their target system, they would fail in their task of depicting a real-life situation. The fiction-view shares similarities with certain literary movements and conceptual models in the sciences but the negative outweigh the positive analogies. This view is not true of scientific models in general.

\section{Conclusion}

The paper has analyzed the fiction-view of scientific modeling and concluded that the disanalogies outweigh the analogies. The analogies show certain similarities between the fiction-view and thought experiments involving demons, on the one hand, and the literary movement of naturalism on the other. But the function of representation shows that scientific modeling is quite different from fiction-writing: it aims at particular target systems; it exploits the interplay between abstraction, idealization, factualization and systematization to great effect; and it is subject to many constraints. The paper has proposed to analyze the notion of representation in terms of 'satisfaction of constraints', as more precise than the notions of 'fit' or 'keys'. Scientific models are about hypothetical worlds, not fictional worlds. 


\section{Acknowledgement}

The author would like to thank two anonymous referees for helpful criticism of an earlier draft.

\section{References}

Bailer-Jones, D., 2003, When Models Represent. International Studies in the Philosophy of Science, 17, 59-74.

--- 2009, Scientific Models in Philosophy of Science. Pittsburgh, University of Pittsburgh Press.

Born, M., 1949, Natural Philosophy of Cause and Chance. Oxford, Clarendon.

Fine, A., 1993, Fictionalism Midwest Studies in Philosophy, XVIII, 1-18.

Giere, R., 2004, How Models are used to represent Reality. Philosophy of Science, 71, 742-752.

--- 2008, Why Models should not be regarded as Works of Fiction. In M. Suárez (ed.): Fiction in Science, London, Routledge, 2009, 248-258.

Godfrey-Smith, P., 2006, The strategy of model-based science. Biology and Philosophy, 21, 725-740.

--- 2009, Models and Fictions in Science. Philosophical Studies, 143, 101-116.

Frigg, R., 2010, Fiction and Scientific Representation. In Roman Frigg and Matthew Hunter (eds.): Beyond Mimesis and Nominalism: Representation in Art and Science, Berlin/New York, Springer 2010, 97-138.

Frigg. R., S. Bradley, H. Du and Leonard A. Smith, 2014, Laplace's Demon and the Adventures of His Apprentices. Philosophy of Science, 81(1), 31-59.

Heilbron, J. L., 1981, Historical Studies in the Theory of Atomic Structure. New York, Arno Press.

Norton, J., 1996, Are Thought Experiments Just What You Thought? Canadian Journal of Philosophy, 26, 333-366.

Pais, A., 1986, Inward Bound. Oxford, Clarendon Press. 
Rutherford, E., 1911, The Scattering of $\mathrm{a}$ and $\mathrm{b}$ Particles by Matter and the Structure of the Atom. Philosophical Magazine, XXI, 669-88.

Suárez, M., 2003, Scientific Representation: Against similarity and isomorphism. International Studies in the Philosophy of Science, 17(3), 225-244.

-- 2004, An inferential conception of scientific representation. Philosophy of Science, 71, 767-779.

--- 2010, Scientific Representation. Philosophy Compass, 5(1), 91-101.

Weinert, F., 1999, Theories, Models and Constraints. Studies in History and Philosophy of Science 30/2, 303-333

--- 2000, The Construction of Atom Models: Eliminative Inductivism and its Relation to Falsificationism. Foundations of Science, 5, 491-531

--- 2006, Einstein and the Representation of Reality. Facta Philosophica, 8, 229-52

--- 2016, The Demons of Science. What they tell us and do not tells us about our world. Springer International Publishing Switzerland. 